\title{
Preserved Endothelium-dependent Vasodilation at the Vasospastic Site in Patients with Variant Angina
}

\author{
Kensuke Egashira, Tetsuzi Inou, Akira Yamada, Yoshitaka Hirooka, and Akira Takeshita \\ The Research Institute of Angiocardiology and Cardiovascular Clinic, Kyushu University Faculty of Medicine, Fukuoka, Japan
}

\begin{abstract}
Endothelial dysfunction has been implicated as a cause of coronary vasospasm in patients with variant angina. This study aimed to determine if endothelium-dependent vasodilation evoked with substance $P$ (SP) was altered at the spastic site where vasospasm was induced by acetylcholine (ACH) in patients with variant angina. It has been shown that SP evokes endothelium-dependent vasodilation with no direct effect on vascular smooth muscle in excised human coronary arteries. SP and ACH were infused into the coronary arteries in nine patients with variant angina in whom coronary arteriograms showed normal or mild atherosclerotic lesions. The vasomotor responses of coronary arteries were assessed by quantitative arteriography. $\mathrm{ACH}$ at a high dose $(100 \mu \mathrm{g} / \mathrm{min})$ provoked coronary vasospasm associated with anginal attack in all patients. In contrast, SP at graded doses $(13.5,40$, and $135 \mathrm{ng} / \mathrm{min})$ caused the dose-dependent and comparable increases in the coronary diameter at the spastic and control sites. ACH at a low dose $(10 \mu \mathrm{g} / \mathrm{min})$ also caused comparable vasodilation at the spastic and control sites in patients with normal coronary arteries. Coronary vasodilating responses to SP were comparable in patients with variant angina and those with atypical chest pain. The results indicate that endothelium-dependent vasodilation evoked with SP and $\mathrm{ACH}$ at the low dose was present at the vasospastic site in patients with variant angina. These findings suggest that the $\mathrm{ACH}$-induced coronary vasospasm in patients with variant angina results from hyperreactivity of vascular smooth muscle to ACH but not from endothelial dysfunction. (J. Clin. Invest. 1992. 89:1047-1052.) Key words: coronary vasospasm • acetylcholine • substance P• endothelium • endothelium-dependent relaxing factor
\end{abstract}

\section{Introduction}

The mechanism of coronary vasospasm remains unknown. It has been suggested that coronary vasospasm results from local nonspecific hyperreactivity to vasoactive stimuli (1-4). Endothelial dysfunction has been implicated as a cause of coronary vasospasm in patients with variant angina, since an intracoronary infusion of acetylcholine at a high dose provokes coronary vasospasm in patients with variant angina $(5,6)$. Coronary va-

Address correspondence to Kensuke Egashira, M.D., Ph.D., The Research Institute of Angiocardiology and Cardiovascular Clinic, Kyushu University School of Medicine, 3-1-1, Maidashi, Higashi-ku, Fukuoka 812, Japan. 1991.

Received for publication 13 June 1991 and in revised form 8 October

J. Clin. Invest.

(c) The American Society for Clinical Investigation, Inc. 0021-9738/92/03/1047/06 \$2.00

Volume 89, March 1992, 1047-1052 sospasm often occurs at the atherosclerotic lesions (1, 2, 7-9), which is associated with defective endothelium-dependent vasodilation $(10,11)$.

However, acetylcholine has the direct vasoconstricting action on vascular smooth muscle in addition to the vasodilating action mediated by endothelium (12-15). Thus, provocation of vasospasm by acetylcholine may result from enhanced vasoconstricting responses of vascular smooth muscle but may not be related to endothelial dysfunction. In fact, the previous studies in our laboratory in miniature pigs have indicated that smooth muscle at the spastic site demonstrates markedly enhanced responses to vasoconstricting agents such as histamine or serotonin (16-20). It also has been shown that atherosclerosis not only impairs endothelium-dependent vasodilation (10, 11 ), but also augments the constrictive responses of vascular smooth muscle to vasoactive agents $(1,2,21)$.

In contrast to acetylcholine, it has been shown that substance $\mathbf{P}$ produces endothelium-dependent vasodilation, but has no direct vasoconstricting action on vascular smooth muscle of human coronary arteries (22-25). Thus, we may assess endothelial function by examining vasomotor responses to substance $P$. It has been shown that substance $P$ causes vasodilation in the in vivo human coronary arteries (26). The purpose of this study was to examine whether endothelium-dependent vasodilation was impaired at the vasospastic site in patients with variant angina. We examined the diameter response to intracoronary substance $\mathbf{P}$ at graded doses at the vasospastic site where vasospasm was provoked by acetylcholine and at the nonspastic control site. We present the results suggesting that endothelial function is present at the spastic site in patients with variant angina.

\section{Methods}

Study patients. Nine patients with variant angina and seven patients with atypical chest pain were studied. All patients with variant angina had a typical history of anginal pain at rest. Seven patients showed transient ST segment elevation during their spontaneous attack, while in two other patients the electrocardiogram during their anginal pain was not recorded. All nine patients with variant angina showed angiographically documented coronary vasospasm associated with anginal pain and ischemic electrocardiographic changes after an intracoronary administration of acetylcholine at a high dose $(100 \mu \mathrm{g} / \mathrm{min})$ (Table I). All patients who were enrolled in this study with a clinical diagnosis of variant angina had vasospasm induced by intracoronary acetylcholine at the high dose. Six patients had angiographically normal coronary arteries and three patients had mild atherosclerotic lesions.

Patients with atypical chest pain had angiographically normal coronary arteries, and responded to intracoronary acetylcholine at the high dose with no evidence of vasospasm or myocardial ischemia. Patients who had myocardial infarction, valvular heart disease or congenital heart disease were excluded from this study.

Written informed consent was obtained from each patient after the study protocol was explained. 
Quantitative coronary arteriography and its analyșis. Coronary cineangiograms were recorded using a Siemens cineangiographic system (Siemens Corp., Iselin, NJ). Non-ionic contrast material (iohexol 350; Dai-ichi Seiyaku Pharmaceutical Corp., Tokyo, Japan) was used. An appropriate view that allowed clear opacification of the study artery with no overlap of other coronary branches was selected. An angle of the view, the distance from $x$-ray focus to the object and that from the object to the image intensifier were carefully kept constant during the study.

The luminal diameter was determined quantitatively by a cinevideodensitometric analysis system (Coronary Analyzer, model XR-70; Vanguard Corp., Melville, NY) (27). An end-diastolic frame of a highquality single-plane angiogram was selected and was acquired into the analyzer. The nontortuous arterial segment of interest was digitized with twofold magnification. Two or more fixed anatomical structures serving as references (branch points) were determined to allow assessment of serial changes in the diameter of the same arterial site. The diameter of the arterial segment of 2-3 mm length was measured at three or more points, and the mean value was used for an analysis. The size of a Judkins catheter was used for calibrating the arterial diameter in millimeters. The inter- and intraobserver reproducibility of the measurements using this quantitative system were high $(r=0.96$ and $r=$ 0.98 , respectively). The percentage changes in the coronary diameter was calculated as follows: (Diameter after drug - baseline diameter/Baseline diameter) $\times 100(\%)$.

It was considered that coronary vasospasm had occurred when the reduction in the luminal diameter by $75 \%$ or more associated with either elevation or depression of ST segment $(>0.1 \mathrm{mV})$ on electrocardiograms was provoked by an intracoronary acetylcholine at a high dose $(100 \mu \mathrm{g})$. In patients with variant angina, we determined changes in the luminal diameter at the spastic and control sites. The control site was defined as an adjacent nonspastic segment proximal or distal to the spastic site when focal spasm was provoked, or as a segment of another nonspastic vessel with the baseline diameter similar to that at the spastic site when diffuse coronary vasospasm was provoked (Table I). The same control site was used for each pair of measurements in the same patient. There was no atherosclerotic lesions found in the control site.

In patients with atypical chest pain, we determined changes in the diameter of the proximal segments of the left anterior descending coronary artery, because in patients with variant angina the acetylcholineinduced coronary vasospasm occurred predominantly in the artery.

Study protocol. Cardiac catheterization was performed in the fasting state after premedication of oral diazepam of $5 \mathrm{mg}$. All antianginal medications such as nitrates and calcium channel blockers were discontinued at least $24 \mathrm{~h}$ before the study. Patients were allowed to use sublingual nitroglycerin if needed, but none of the patients had used nitroglycerin within an hour before catheterization.

After completion of baseline coronary arteriography by a femoral approach, the following drugs were infused in patients with variant angina and those with atypical chest pain. First, physiological saline was gradually infused into the left and right coronary artery through the Judkins catheter. Coronary arteriography was performed 2 min after the saline infusion. Second, substance $P$ at graded doses of $13.5,40$, and $135 \mathrm{ng}$ were infused over $1 \mathrm{~min}$ into the left coronary artery and then into the right coronary artery. Coronary arteriography was performed 2 min after each dose of substance $P$ when arterial pressure had returned to the baseline value. The intracoronary infusion of saline or substance $\mathrm{P}$ was performed manually at a rate of $\sim 1.0-1.2 \mathrm{ml} / 10 \mathrm{~s}$ and the total volume of infusion was $5 \mathrm{ml}$. We waited for $2 \mathrm{~min}$ before infusing the next dose of substance $P$. Third, acetylcholine at a high dose (100 $\mu \mathrm{g} /$ min) was administered into the left coronary artery and then into the right coronary artery to provoke coronary vasospasm. Coronary angiograms were recorded $1 \mathrm{~min}$ after the injection of acetylcholine when hemodynamic parameters returned to the baseline level. The studies with acetylcholine were done while a $5 \mathrm{~F}$ pacing catheter was placed in the right ventricle and the pacing rate was set in the demand mode at 50 beats/min. When anginal pain or vasospasm persisted for $2 \mathrm{~min}$ or more, isosorbide dinitrate $2 \mathrm{mg}$ was administered into the spastic ar- tery. After coronary vasospasm was relieved either spontaneously or by isosorbide dinitrate, we waited at least for $20 \mathrm{~min}$, by which time the coronary diameter had returned nearly to the baseline level.

Finally, in order to test the endothelium-related vasodilation mediated by acetylcholine at the vasospastic site, acetylcholine at a low dose ( $10 \mu \mathrm{g} / \mathrm{min}$ for $2 \mathrm{~min}, 0.5 \mathrm{ml} / \mathrm{min}$ ) was infused selectively into the left anterior descending artery of seven patients with variant angina (Nos. 1-7, Table I) and then coronary arteriography was performed. The subselective infusion of the drug was done through a $3 \mathrm{~F}$ catheter that was introduced into the proximal portion of the artery, because infusion of acetylcholine into the circumflex or right coronary artery results in bradyarrhythmias and hypotension which altered the druginduced vasomotion.

At the end of the study, isosorbide dinitrate of $2 \mathrm{mg}$ was administered into the left and right coronary arteries in all patients, and coronary angiograms of multiple projections were recorded to assess the severity of atherosclerotic lesions. Phasic and mean systolic arterial pressure, heart rate and standard 12-leads electrocardiograms were continuously monitored using a Nihon-Kohden Polygraph System (Nihon Kohden Inc., Tokyo, Japan) and were recorded on a multi-channel recorder.

Preparation of substance $P$ and acetylcholine. Substance $P$ (Sigma Chemical Co., St. Louis, MO) of $0.1 \mathrm{mM}$ in $10 \%$ acetic acid was prepared and stored at $0^{\circ} \mathrm{C}$ until use. Acetylcholine chloride (Dai-ichi Seiyaku Pharmaceutical Corp.) of $2 \mathrm{mg}$ was freeze-dried and stored at $0^{\circ} \mathrm{C}$. Further dilutions were made with physiological saline immediately before use.

Statistical analysis. Data are expressed as mean \pm SE. Student's $t$ test was used for comparisons of paired or unpaired data. When serial changes in the coronary diameter or hemodynamic variables in response to substance $P$ were compared, analysis of variance (ANOVA) ${ }^{1}$ for repeated measures followed by a Bonferroni's multiple comparison test was used (28). Comparisons of hemodynamic parameters and coronary vasomotion between patients with variant angina and those with atypical chest pain were done using a two-way ANOVA. Probability of less than 0.05 was considered statistically significant.

\section{Results}

Provocation of coronary vasospasm. In patients with atypical chest pain, neither vasospasm, anginal pain nor ischemic electrocardiographic changes occurred after intracoronary acetylcholine at the high dose. The percentage change in the diameter of the proximal left anterior descending coronary artery evoked with acetylcholine was $-22 \pm 9 \%$.

An intracoronary administration of acetylcholine at the high dose $(100 \mu \mathrm{g} / \mathrm{min})$ provoked coronary vasospasm at one or more coronary arteries in all nine patients with variant angina (Table I). Coronary vasospasm occurred at only the left anterior descending artery in five patients, at the left anterior descending artery and left circumflex artery in two patients, and at the left anterior descending artery and right coronary artery in two patients. Thus, the acetylcholine-induced coronary vasospasm occurred at 13 coronary arteries in 9 patients. The percentage change in the diameter induced by acetylcholine was $-94 \pm 2$ in the spastic site and $-18 \pm 5 \%$ in the control site $(P<0.01)$.

Responses to substance $P$ at graded doses. Changes in hemodynamic variables during infusion of saline or substance $P$ are summarized in Table II. Intracoronary infusion of saline had little effects on arterial pressure, heart rate and the coronary diameters. Mean arterial pressure decreased transiently after

1. Abbreviation used in this paper: ANOVA, analysis of variance. 
Table I. Clinical Characteristics of the Patients

\begin{tabular}{|c|c|c|c|c|c|c|c|c|}
\hline \multirow[b]{2}{*}{ No. } & \multirow[b]{2}{*}{ Age } & \multirow[b]{2}{*}{ Sex } & \multicolumn{3}{|c|}{ Coronary arteriographic findings } & \multirow[b]{2}{*}{ Vasospastic site } & \multirow[b]{2}{*}{ Control site } & \multirow{2}{*}{$\begin{array}{l}\text { ST segment changes } \\
\text { during the acetylcholine- } \\
\text { induced anginal pain }\end{array}$} \\
\hline & & & LAD & LCX & RCA & & & \\
\hline 1 & 37 & $\mathbf{M}$ & $\mathbf{N}$ & $\mathbf{N}$ & $\mathbf{N}$ & LADseg-7 & seg-6 & Depression in V3-6 \\
\hline \multirow[t]{2}{*}{2} & 61 & $\mathbf{F}$ & $\mathbf{N}$ & $\mathbf{N}$ & $\mathbf{N}$ & LADseg-7 \& 8 & seg-6 & Depression in II, III, aVf \\
\hline & & & & & & RCAseg-1 \& 3 & seg-1 & \\
\hline 3 & 52 & $\mathbf{M}$ & $\mathbf{N}$ & $\mathrm{N}$ & $\mathbf{N}$ & LADseg-8, $9 \& 10$ & seg- 6 & Elevation in V5-6, II, III, aVf \\
\hline 4 & 38 & $\mathbf{M}$ & $\mathbf{N}$ & $\mathbf{N}$ & $\mathbf{N}$ & LADseg-7 \& 9 & seg-6 & Elevation in V2-5 \\
\hline \multirow[t]{2}{*}{5} & 52 & $\mathbf{M}$ & $\mathrm{N}$ & $\mathbf{N}$ & $\mathbf{N}$ & LADseg-7 & seg-6 & Elevation in V3-6 \\
\hline & & & & & & RCAseg-2 & seg-1 & Elevation in II, III, aVf \\
\hline \multirow[t]{2}{*}{6} & 58 & $\mathbf{M}$ & seg-6:50\% & $\mathbf{N}$ & $\mathbf{N}$ & LADseg-6 (distal) & seg-6 (proximal) & Elevation in V2-6 \\
\hline & & & & & & LCX seg-11 & $\operatorname{seg}-3$ & Depression in II, III, aVf \\
\hline 7 & 62 & $\mathbf{M}$ & seg-7:41\% & $\mathrm{N}$ & seg-1:27\% & LADseg-7 & seg-6 & Elevation in V3-6 \\
\hline 8 & 48 & $\mathbf{M}$ & seg- $7: 25 \%$ & seg-12:33\% & 4PD:44\% & LADseg-7 & seg-6 & $\begin{array}{l}\text { Elevation in V3-5 and } \\
\text { Depression in II, III and aVf }\end{array}$ \\
\hline \multirow[t]{2}{*}{9} & 58 & $\mathbf{M}$ & $\mathbf{N}$ & $\mathrm{N}$ & $\mathbf{N}$ & LADseg-7 & seg-6 & Elevation in V5-6 \\
\hline & & & & & & LCXseg-12 & seg-11 & Elevation in II, III, aVf \\
\hline
\end{tabular}

The location of coronary atherosclerotic lesions or vasospasm is denoted according to the definition of American Heart Association (AHA committee report. 1975. A reporting system on patients evaluated for coronary artery disease. Circulation 51:7). The severity of coronary atherosclerotic lesions is assessed by coronary angiograms after isosorbide dinitrate. $\mathrm{ACH}$, acetylcholine; $\mathrm{M}$, male, $\mathrm{F}$, female; $\mathrm{N}$, normal coronary artery; LAD, the left anterior descending coronary artery; LCX, the left circumflex coronary artery; RCA, the right coronary artery.

each dose of substance $\mathbf{P}$ (data are not shown), but it returned to the baseline level by $2 \mathrm{~min}$ after the infusion when coronary arteriography was performed. Heart rate did not significantly change after the doses of 13.5 and $40 \mathrm{ng} / \mathrm{min}$, but it signifcantly increased after the dose of $135 \mathrm{ng} / \mathrm{min}$.

Fig. 1 shows serial angiograms in a patient with variant angina, which denotes that acetylcholine at the high dose induced coronary vasospasm at the left anterior descending coronary artery (Fig. $1 C$ ), whereas substance $\mathrm{P}$ dilated coronary arteries at the spastic site as well as the control site (Fig. $1 B$ ). Changes in the coronary diameters in response to substance $P$ are summarized in Table II and Fig. 2. The baseline diameter in the spastic site did not differ from that in the control site. Substance $P$ increased the coronary diameters at the spastic and control sites in a dose-dependent manner. The magnitudes of coronary dilation evoked with substance $P$ at graded doses were comparable between the spastic and control sites: the percentage increases in the diameter in response to substance $P$ at the doses of $13.5,40$, and $135 \mathrm{ng} / \mathrm{min}$ were $12 \pm 3,19 \pm 5$, and $28 \pm 6 \%$ in the spastic site, and $7 \pm 3,18 \pm 4$, and $27 \pm 5 \%$ in the control site, respectively.

The magnitudes of coronary vasodilation evoked with substance $P$ at the graded doses did not differ in patients with atypical chest pain and those with variant angina (by ANOVA) (Table II, Fig. 2).

Response to acetylcholine at a low dose. Infusion of acetylcholine at the low dose $(10 \mu \mathrm{g} / \mathrm{min}$ for $2 \mathrm{~min})$ into the left anterior descending coronary artery was performed in seven patients with variant angina (Table I, No. 1-7). The infusion of acetylcholine at the low dose was not done in the other two

Table II. Changes in Hemodynamic Variables during Infusion of Saline or Substance P

\begin{tabular}{|c|c|c|c|c|c|}
\hline & \multirow[b]{2}{*}{ Baseline } & \multirow[b]{2}{*}{$\begin{array}{l}\text { Physiological } \\
\text { saline }\end{array}$} & \multicolumn{3}{|c|}{ Substance $\mathbf{P}(\mathrm{ng} / \mathrm{min})$} \\
\hline & & & 13.5 & 40 & 135 \\
\hline \multicolumn{6}{|l|}{ Patients with variant angina } \\
\hline Mean arterial pressure $(\mathrm{mmHg})$ & $88 \pm 3$ & $91 \pm 3$ & $92 \pm 3$ & $85 \pm 4$ & $85 \pm 3$ \\
\hline Heart rate (beats/min) & $67 \pm 4$ & $65 \pm 4$ & $66 \pm 5$ & $69 \pm 5$ & $73 \pm 4^{*}$ \\
\hline \multicolumn{6}{|l|}{ Coronary artery diameter $(\mathrm{mm})$} \\
\hline the spastic site & $2.3 \pm 0.2$ & $2.3 \pm 0.2$ & $2.4 \pm 0.2^{*}$ & $2.6 \pm 0.2^{*}$ & $2.9 \pm 0.2 \ddagger$ \\
\hline the control site & $2.3 \pm 0.2$ & $2.4 \pm 0.2$ & $2.5 \pm 0.2^{*}$ & $2.8 \pm 0.2 \ddagger$ & $2.9 \pm 0.2 \ddagger$ \\
\hline \multicolumn{6}{|l|}{ Patients with atypical chest pain } \\
\hline Mean arterial pressure $(\mathrm{mmHg})$ & $82 \pm 2$ & $82 \pm 3$ & $81 \pm 3$ & $80 \pm 3$ & $84 \pm 3$ \\
\hline Heart rate (beats/min) & $68 \pm 5$ & $66 \pm 4$ & $66 \pm 5$ & $68 \pm 4$ & $79 \pm 6^{*}$ \\
\hline Coronary artery diameter $(\mathrm{mm})$ & $2.5 \pm 0.4$ & $2.5 \pm 0.4$ & $2.7 \pm 0.4^{*}$ & $2.9 \pm 0.3 \ddagger$ & $3.1 \pm 0.4 \ddagger$ \\
\hline
\end{tabular}

Data are mean \pm SEM.

${ }^{*} P<0.05,{ }^{\ddagger} P<0.01$ versus baseline value by ANOVA followed by a Bonferroni’s multiple comparison test. There was no significant difference in responses of hemodynamic parameters and the diameter of coronary artery evoked with substance $P$ between patients with variant angina and those with atypical chest pain (by ANOVA). 

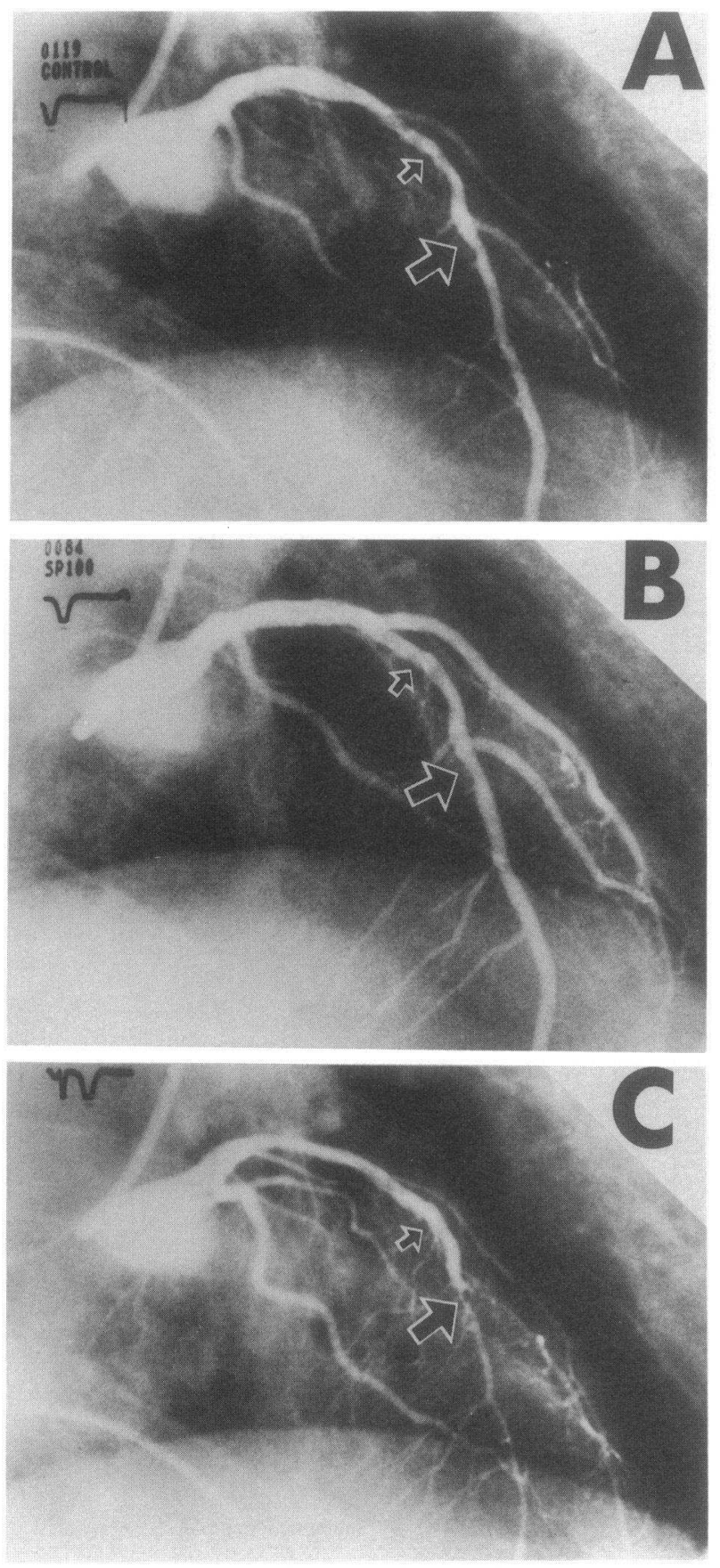

Figure 1. Serial coronary angiograms of the left coronary arteries in a patients (the right anterior oblique view). A baseline angiogram is shown in $(A)$. An intracoronary infusion of substance $\mathrm{P}(135 \mathrm{ng} / \mathrm{min})$ increased the diameters of all coronary branches $(B)$. In contrast, acetylcholine $(100 \mu \mathrm{g} / \mathrm{min})$ provoked coronary vasospasm at the distal left anterior descending artery and diagonal branches $(C)$, which was accompanied by anginal pain and ischemic ST segment elevation in V5-6, II, III and aVf leads. The large and small arrows denote the spastic and the control sites, respectively.

patients, because large doses of isosorbide dinitrate $(5 \mathrm{mg}$ or more) were required to relieve the coronary vasospasm in these two patients, and thus hemodynamic parameters such as arterial pressure and the diameter of the coronary artery did not return to the baseline level after we waited for $30 \mathrm{~min}$ or more.

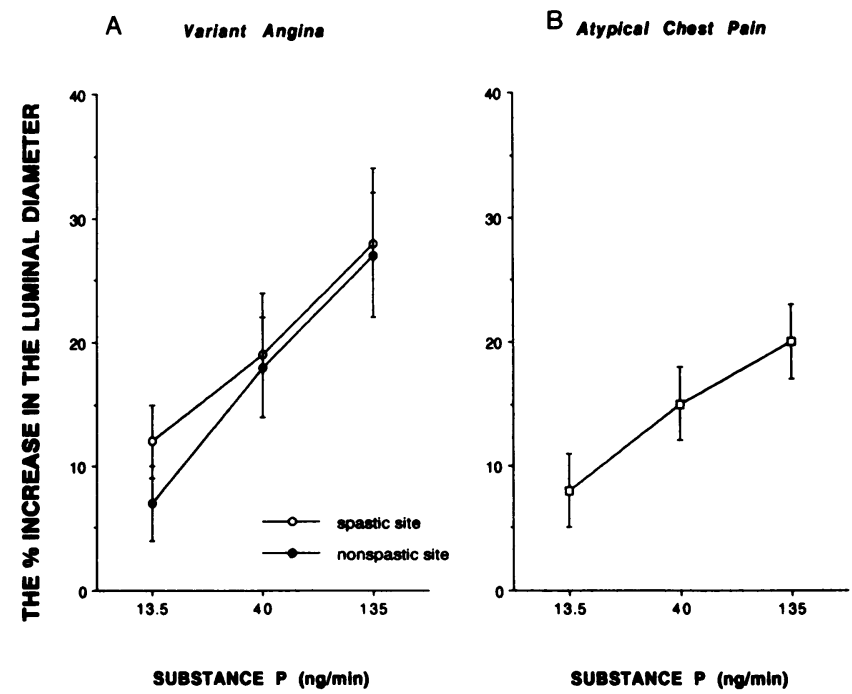

Figure 2. The vasodilating responses to substance $\mathbf{P}$ (the percentage increase in the luminal diameter from the baseline value) at the spastic (open circles) and control (closed circles) site in patients with variant angina $(A)$, and those at the left anterior descending coronary artery in patients with atypical chest pain and normal coronary arteries $(B)$.

Arterial pressure and heart rate were stable during infusion of acetylcholine at the low dose. In five patients in whom the study artery was angiographically normal (Table I, No. 1-5), acetylcholine caused comparable vasodilation at the spastic site $(7 \pm 1 \%)$ and control site $(7 \pm 2 \%)$. In contrast, acetylcholine at the low dose caused modest vasoconstriction at both the spastic and control sites in two patients in whom the study artery had mild atherosclerotic lesions (Table I, No. 6-7). There was a close correlation $(r=0.92, P<0.001)$ in the diameter responses of the spastic and control site to acetylcholine at the low dose (Fig. 3), which indicates that vasomotor responses to acetylcholine at the low dose were similar between the spastic and control sites irrespective of vasodilating or vasoconstricting response.

Response to isosorbide dinitrate. In patients with variant angina, an intracoronary administration of isosorbide dinitrate of $2 \mathrm{mg}$ increased the coronary diameter from $2.2 \pm 0.2$ to $3.0 \pm 0.2 \mathrm{~mm}(P<0.01)$ at the spastic site, and from $2.3 \pm 0.2$ to $3.1 \pm 0.2 \mathrm{~mm}(P<0.01)$ at the control site. The percentage changes in the diameter at the spastic site $(34 \pm 5 \%)$ did not
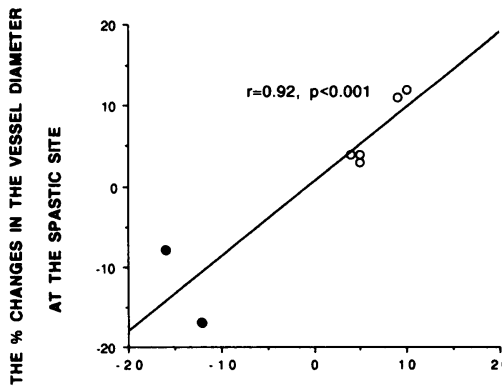

the \% changes in the vessel diameter AT THE CONTROL STTE
Figure 3. A scatter plot of the percentage changes in the diameter at the spastic and control site of coronary artery in each patient in response to acetylcholine at a low dose $(10$ $\mu \mathrm{g} / \mathrm{min}$ for $2 \mathrm{~min}$ ). Open circles denote the responses of normal coronary arteries, and closed circles denote coronary arteries with mild atherosclerotic lesions. There is a positive linear correlation $(r$ $=0.92, P<0.001)$ in the responses at the spastic and the control sites. 
significantly differ from that at the control site $(32 \pm 6 \%)$. Isosorbide dinitrate significantly $(P<0.01)$ increased the coronary artery diameter of patients with atypical chest pain from $2.6 \pm 0.4$ to $3.3 \pm 0.4 \mathrm{~mm}(25 \pm 6 \%$ dilation $)$.

\section{Discussion}

The major finding of this study is that substance P produced significant vasodilation at the spastic site where coronary vasospasm was provoked by acetylcholine at the high dose (100 $\mu \mathrm{g} / \mathrm{min})$. The magnitudes of coronary vasodilation evoked with substance $P$ at graded doses were comparable between the spastic and control sites. These results suggest that the vasodilating response to substance $P$ did not differ between the spastic and nonspastic sites in patients with variant angina.

We infused substance $P$ at graded doses to examine endothelial function of coronary artery, because it has been shown that substance $P$ evokes endothelium-dependent vasodilation with no direct action on vascular smooth muscles in excised human coronary arteries (22-25). In this respect, substance $P$ differs from acetylcholine which has the dual effects: the endothelium-dependent vasodilation and the direct contraction of vascular smooth muscle (12-15). As shown previously (26), substance $P$ produced dilation of the epicardial coronary arteries in a dose-dependent manner in our patients. The magnitudes of coronary vasodilation evoked with substance $P 135 \mathrm{ng} / \mathrm{min}$ were comparable with those evoked with isosorbide dinitrate 2 $\mathrm{mg}$. An important finding of this study is that the magnitudes of coronary vasodilation evoked with substance $P$ were comparable between the spastic and control site. The results indicate that endothelium-dependent vasodilation evoked with substance $P$ was present at the spastic site, and was comparable at the spastic and control site.

We considered the possibility that there may be a selective impairment of endothelium-dependent vasodilation mediated by acetylcholine at the spastic sites, since vasospasm was provoked by acetylcholine at the high dose. Thus, we examined the response to acetylcholine at the low dose $(10 \mu \mathrm{g} / \mathrm{min}$ for $2 \mathrm{~min})$. This dosage of acetylcholine has been used to examine endothelium-dependent vasodilation mediated by acetylcholine in humans $(5,29,30)$. In the present study, acetylcholine at the low dose dilated coronary artery at the spastic and control sites in patients with no atherosclerotic coronary lesions, and the magnitudes of coronary vasodilation evoked with acetylcholine at the low dose were comparable between the two sites. Thus, acetylcholine at the high dose provoked coronary vasospasm, but acetylcholine at the low dose caused comparable vasodilation at the spastic and control sites. These results suggest that endothelium-dependent vasodilation mediated by acetylcholine was present at the vasospastic site, but was overcome by augmented direct vasoconstriction of the smooth muscle when acetylcholine at the high dose was applied. In two patients with mild atherosclerotic lesions at the spastic site, acetylcholine at the low dose constricted both the spastic and control site, but the magnitudes of vasoconstriction were comparable between the two sites. Thus, defective endothelium-dependent vasodilation with acetylcholine at the low dose was not specific to the spastic site. It has been shown that endothelium-dependent vasodilation evoked with acetylcholine is impaired in the artery with even mild atherosclerotic lesions $(5,29)$ or with the presence of coronary risk factors such as hypercholesterolemia (30,
31). The latter findings may account for the results in our patients with mild atherosclerotic lesions. Thus, our results indicate that endothelium-dependent vasodilation mediated by acetylcholine at the spastic site might not differ from that at the control site.

Endothelial dysfunction has been implicated as a cause of coronary vasospasm in patients with variant angina $(1,2,5,6)$. This suggestion is based mostly on the fact that an intracoronary administration of acetylcholine provoked coronary vasospasm in patients with variant angina (6), while this compound caused modest constriction of atherosclerotic but nonspastic coronary artery and dilated the angiographically normal coronary arteries $(5,29-31)$. However, the striking vasospastic response to acetylcholine at the high dose in our patients with variant angina is not typical of normal or atherosclerotic coronary arteries in patients with no evidence of variant angina. A recent preliminary report by Yamamoto and co-workers has suggested that coronary vasodilation evoked with substance $P$ at a dose of $20 \mathrm{pmol} / \mathrm{min}(27 \mathrm{ng} / \mathrm{min})$ was comparable between the site of vasospasm induced by ergonovine maleate and the control site (32). Our findings agree with their results and further suggest that coronary vasodilation evoked with substance $\mathrm{P}$ at graded doses as well as with acetylcholine at the low dose did not differ between the site of coronary vasospasm induced by acetylcholine at the high dose and the control site. These results suggest not only that endothelial dysfunction is not prerequisite to coronary vasospasm, but also that acetylcholine-induced vasospasm results primarily from augmented vasoconstriction of vascular smooth muscle to acetylcholine. The results are consistent with our previous works in miniature pigs with coronary artery spasm (16-20). In this animal model of coronary vasospasm, vascular smooth muscle from the spastic segments show markedly augmented responses to histamine and serotonin as compared to those of the nonspastic segments.

It has been shown that even mild atherosclerosis impairs endothelium-dependent vasodilation (29-31). It is possible, therefore, that the vasodilating response to substance $\mathrm{P}$ might be blunted in both the vasospastic site and control site in our patients. However, this is unlikely because coronary vasodilation induced by substance $P$ in patients with atypical chest pain and normal coronary arteries did not differ from that in patients with variant angina (Fig. 2).

In conclusion, this study has demonstrated that substance $P$ at graded doses caused the dose-dependent and comparable coronary vasodilation at the spastic and control sites in patients with variant angina. These findings suggest that endothelial function is present at the site of acetylcholine-induced coronary vasospasm in patients with variant angina. Although we can not exclude the possibility that endothelial dysfunction may play a role in spontaneously occurring coronary vasospasm, the results of the present study suggest that acetylcholine-induced coronary vasospasm in patients with variant angina results primarily from hyperreactivity of vascular smooth muscle to acetylcholine, but not from local endothelial dysfunction. It is still possible, however, that endothelial dysfunction may play some role in promoting vasospasm in patients with and without variant angina.

\section{Acknowledgments}

This study was supported in part by grant-in-aid for Scientific Research 02404045 and 02454259 , and by grant-in-aid for Scientific Research 
on Priority Areas 03268226 from the Ministry of Education, Science and Culture, Tokyo, Japan.

\section{References}

1. Maseri, A., G. Davies, D. Hackett, and J. C. Kaski. 1990. Coronary artery spasm and vasoconstriction. The case of distinction. Circulation. 81:1983-1991.

2. Ganz, P., and R. W. Alexander. 1985. New insights into the cellular mechanisms of coronary vasospasm. Am. J. Cardiol. 56:11E-15E.

3. Kaski, J. C., F. Crea, D. Meran, L. Rodrigues, S. Chierchia, G. Davies, and A. Maseri. 1986. Local coronary supersensitivity to diverse vasoconstrictor stimuli in variant angina. Circulation. 74:1255-1265.

4. Waters, D. D., J. Szlachcic, R. Bonan, D. D. Miller, F. Dauwe, and P. Theroux. 1983. Comparable sensitivity of exercise, cold pressure and ergonovine testing on provoking attacks of variant angina in patients with active disease. Circulation. 67:310-315.

5. Ludmer, P. L., A. P. Selwyn, T. L. Shook, R. R. Wayne, G. H. Mudge, R. W. Alexander, and P. Ganz. 1986. Paradoxical vasoconstriction induced by acetylchilone in atherosclerotic coronary arteries. N. Engl. J. Med. 315:10461051.

6. Yasue, H., Y. Horio, N. Nakamura, H. Fujii, N. Imoto, R. Sonoda, K. Kugiyama, K. Obata, Y. Morikami, and T. Kimura. 1986. Induction of coronary artery spasm by acetylcholine in patients with variant angina: possible role of the parasympathetic nervous system in the pathogenesis of coronary artery spasm. Circulation. 74:955-963.

7. Hillis, L. D., and E. Braunwald. 1978. Coronary artery spasm. N. Engl. J. Med. 299:695-702.

8. Shepherd, J. T., and P. M. Vanhoutte. 1985. Spasm of the coronary arteries: Cause and consequence (the scientist's view point). Mayo. Clin. Proc. 60:33-46.

9. Nakamura M. 1985. Myocardial Ischemia (President lecture). Jpn. Circ. J. 49:1-12

10. Verbeuren, T. J., F. H. Jordaens, L. L. Zonnekeyn, C. E. VanHove, M. C Coene, and A. G. Herman. 1986. Effects of hypercholesterolemia on the vascular reactivity in the rabbit. Circ. Res. 58:552-564.

11. Freiman, P. C., G. G. Mitchell, D. D. Heistad, M. L. Armstrong, and D. G. Harrison. 1986. Atherosclerosis impairs endothelium-dependent vascular relaxation to acetylcholine and thrombin in primates. Cir. Res. 58:783-789.

12. Furchgott, R. F. 1983. Role of endothelium in response of vascular smooth muscle. Circ. Res. 53:557-573.

13. Bassenge, E., and R. Busse. 1988. Endothelial modulation of coronary tone. Prog. Cardiovasc. Dis. 30:349-380.

14. Lücher, T. F., V. Richard, M. Tschudi, Z. Yang, and C. Boulanger. 1990 Endothelial control of vascular tone in large and small coronary arteries. J. Am. Coll. Cardiol. 15:512-527.

15. Kalsner, S. 1985. Cholinergic mechanisms in human coronary artery preparation. Implications of species difference. J. Physiol. (Lond.). 358:509-516.

16. Egashira, K., H. Tomoike, Y. Yamamoto, A. Yamada, Y. Hayashi, and M. Nakamura. 1986. Histamine-induced coronary spasm in regions of intima thickening in miniature pigs. Roles of cholesterol and spontaneous or induced intimal thickening. Circulation. 74:826-837.
17. Egashira, K., H. Shimokawa, H. Tomoike, and M. Nakamura. 1987. Effects of prostanoid on coronary circulation and coronary artery spasm. Jpn. Circ. J. 51:459-461.

18. Yamomoto, Y., H. Tomoike, K. Egashira, and M. Nakamura. 1987. Attenuation of endothelium-related relaxation and enhanced responsiveness of vascular smooth muscle in spastic coronary artery segments from miniature pigs. Circ. Res. 61:772-778.

19. Yamamoto, Y., H. Tomoike, K. Egashira, T. Kobayashi, K. Kawasaki, and M. Nakamura. 1987. Pathogenesis of coronary artery spasm in miniature swine with regional intimal thickening after balloon denudation. Circ. Res. 60:113-121.

20. Satoh, S., H. Tomoike, W. Mitsuoka, S. Egashira, H. Tagawa, T. Kuga, and M. Nakamura. 1990. Smooth muscles from spastic coronary artery segments show hypercontractility to histamine. Am. J. Physiol. 259 (Heart. Circ. Physiol. 28):H9-H13.

21. Heistad, D. D., M. L. Armstrong, M. L. Marcus, D. J. Piegors, and A. L. Mark. 1984. Augmented responses to vasoconstrictor stimuli in hypercholesterolemic and atherosclerotic monkeys. Circ. Res. 54:711-718.

22. Bossaller, C., G. B. Habib, H. Yamamoto, C. Williams, S. Wells, and P. D. Henry. 1987. Impaired muscarinic endothelium-dependent relaxation and cyclic guanosine 5 '-monophosphate formation in atherosclerotic human coronary artery and rabbit aorta. J. Clin. Invest. 79:170-174.

23. Toda, N., and T. Okamura. 1989. Endothelium-dependent and independent responses to vasoactive substances of isolated human coronary arteries. Am. J. Physiol. 257 (Heart and Circ. Physiol. 26):H988-995.

24. Chester, A. H., G. S. O'neil, S. Moncada, S. Tadjkarimi, and M. Yacoub. 1990. Low basal and stimulated release of nitric oxide in atherosclerotic epicardial coronary arteries. Lancet. 336:897-900.

25. Förstermann, U., A. Mügge, U. Alheim, A. Haverich, and J. C. Frölich 1988. Selective attenuation of endothelium-mediated vasodilation in atherosclerotic human coronary arteries. Circ. Res. 62:185-190.

26. Crossman, D. C., S. W. Larkin, R. W. Fuller, G. J. Davies, and A. Maseri.

1989. Substance $P$ dilates epicardial coronary arteries and increases coronary blood flow in humans. Circulation. 80:475-484.

27. Nichols, A. B., C. F. O. Gabrieli, J. J. Fenoglio, and P. D. Esser. 1984 Quantification of relative coronary arterial stenosis by videodensitometric analysis of the coronary angiograms. Circulation. 69:512-522.

28. Glantz, S. A., and B. K. Slinker. 1990. Primer of Applied Regression and Analysis of Variance. McGraw-Hill, New York. 272-380.

29. Werns, S. W., J. A. Walton, H. H. Hsia, E. G. Nabel, M. L. Sanz, and B. Pitt. 1989. Evidence of endothelial dysfunction in angiographically normal coronary arteries of patients with coronary artery disease. Circulation. 79:287-291.

30. Vita, J. A., C. B. Treasure, E. G. Nabel, J. M. McLenachan, R. D. Fish A. C. Yeung, V. I. Vekshtein, A. P. Selwyn, and P. Ganz. 1990. Coronary vasomotor response to acetylcholine relates to risk factors for coronary artery disease. Circulation. 81:491-497.

31. Zeiher, A. M., H. Drexler, H. Wollschläger, and H. Just. 1991. Modulation of coronary vascular tone in humans. Progressive endothelial dysfunction with different early stages of coronary atherosclerosis. Circulation. 83:391-401.

32. Yamamoto, H., H. Yoshimura, M. Noma, H. Kai, S. Suzuki, T. Tajimi, M. Sugihara, and Y. Kikuchi. 1990. Evaluation of endothelial function of spastic segment in patients with variant angina. Circulation. 82(Suppl. 3):421 (Abstr.) 